\title{
Space Application of Piezoelectric Wafer Active Sensors for Structural Health Monitoring**
}

\author{
V. Giurgiutiu, * B. Lin, G. SAntoni-Bottai And A. CuC \\ University of South Carolina, Columbia, SC 29208, USA
}

\begin{abstract}
Piezoelectric wafer active sensors (PWAS) are lightweight and inexpensive enablers for a large class of structural health monitoring (SHM) applications. This paper presents and discusses the challenges and opportunities related to the use of PWAS in the structures specific to space applications. The challenges posed by space structures are often different from those encountered in conventional structures. After a review of PWAS principles, the paper discusses the multi-physics power and energy transduction between structurally guided waves and PWAS; predictive modeling results using a simplified analytical approach are presented. Experimental results on space-like specimen structures are presented. Survivability of PWAS transducers under cryogenic space-like conditions is experimentally verified. The paper ends with conclusions and suggestions for further work.
\end{abstract}

Key Words: structural health monitoring, piezoelectric, sensors, PWAS, SHM, space applications, satellites, space vehicles, international space station, orbital plane.

\section{INTRODUCTION}

$\mathbf{S}$ TRUCTURAL health monitoring (SHM) is an emerging technology with multiple applications in the evaluation of critical structures. The goal of SHM research is to develop a monitoring methodology that is capable of detecting and identifying, with minimal human intervention, various damage types during the service life of the structure. Numerous approaches have been utilized in recent years to perform structural health monitoring; they can be broadly classified into two categories: passive methods and active methods. Passive SHM methods (such as acoustic emission, impact detection, strain measurement, etc.) have been studied longer and are relatively mature; however, they suffer from several drawbacks which limit their utility (need for continuous monitoring, indirect inference of damage existence, etc.). Active SHM methods are currently of greater interest due to their ability to interrogate a structure at will. One of the promising active SHM methods utilizes arrays of piezoelectric wafer active sensors (PWAS) bonded to a structure for both transmitting and receiving ultrasonic waves in order to achieve damage detection (Giurgiutiu, 2008). In thin-wall structures, PWAS are effective guided

\footnotetext{
**Manuscript based on paper SMASIS2010-3811 presented at ASME2010 Conference on Smart Materials, Adaptive Structures and Intelligent Systems, SMASIS 2010, Sept. 28 to Oct. 1, 2010, Philadelphia, PA

*Author to whom correspondence should be addressed.

E-mail: victorg@sc.edu

Figures 1-11 appear in color online: http://jim.sagepub.com
}

wave transducers by coupling their in-plane motion with the guided wave particle motion on the material surface. The in-plane PWAS motion is excited by the applied oscillatory voltage through the $d_{31}$ piezoelectric coupling. Optimum excitation and detection happens when the PWAS length is in certain ratios with the wavelength of the guided wave modes. The PWAS action as ultrasonic transducers is fundamentally different from that of conventional ultrasonic transducers. Conventional ultrasonic transducers act through surface tapping, that is, by applying vibration pressure to the structural surface. The PWAS transducers are physically bonded to the structure and act through surface pinching, and are strain coupled with the structural surface. This allows the PWAS transducers to have a greater efficiency in transmitting and receiving ultrasonic surface and guided waves when compared with the conventional ultrasonic transducers.

This article presents and discusses the challenges and opportunities related to the use of PWAS in structures specific to space applications. The paper starts with a brief presentation of the challenges posed by space structures, which are often different from those encountered in conventional structures. Then, it reviews the principles of PWAS-based SHM. Subsequently, the paper discusses the analytical challenges of studying the multi-physics power and energy transduction between structurally guided waves and PWAS. Predictive modeling results using a simplified analytical approach are presented and discussed. Experimental results on using PWAS technology to detect damage in space-like specimen structures are 
presented. The operability and survivability of PWAS transducers under cryogenic space-like conditions are verified. The paper ends with conclusions and suggestions for further work.

\section{SPACE STRUCTURES SHM NEEDS}

The SHM of space structures encompasses a larger spectrum of utilization than traditional SHM applications. In traditional SHM applications, the SHM methodology is usually aimed at detecting in-service damage and degradation thus facilitating the transition from scheduled-based maintenance to needs-based maintenance. This application of the SHM concept offers the potential of saving time, reducing maintenance costs, avoiding unnecessary down time, as well as increasing safety and availability.

For space structures, the use of the SHM concept could be more extensive. On the one hand, maintenance-focused SHM could be applied to some space applications such as international space station, $\mathrm{X}-37$ demonstrator, future space shuttle, orbital space plane, etc. For such applications, the SHM system would monitor structural anomalies such as cracks and impact damage (Figure 1(a)). Corrosion could also be an issue, because inhabited space vehicles that have to sustain a human environment may experience atmospheric condensation and drainage leaks. On the other hand, some space SHM applications may not have maintenance as the main focus. For example, the responsive space initiative aims at rapidly providing space capabilities tailored to the immediate needs of the warfighter in the field (Sega and Cartwright, 2007). In a 6-day window, a satellite solution to a tactical situation should be designed, built from stocked components, tested, integrated, launched, checked-out on orbit, and delivering data directly to the warfighter. This implies that the typical testing protocol performed to assess structural surety before space launch must be severely truncated (Sarafin and Doukas,
2007). A responsive space satellite (Figure 1(b)) would likely consist of modular panels tightly packed with electronics embedded into the structure. New rapid methods are being developed by Air Force Research Laboratories, Space Vehicles Directorate (AFRL/ RVSV) at Kirtland AFB to assess the surety of the satellite structure within tight timelines (Arritt et al., 2007, 2008). One approach is to interrogate it with an array of structural sensors that would be able to detect structural flaws (delaminated panels, bolts not torqued correctly, etc.). Chang's group at Stanford University has studied the detection of bolt loosening using wave transmission between piezoelectric sensors (Yang and Chang, 2006; Xie et al., 2007). Lanza di Scalea's group at UC San Diego has used active ultrasonic techniques to evaluate joint integrity (Clayton et al., 2008). Doyle et al. (2008, 2009) have used several active-sensing techniques for assessing bolted joint integrity such as the acousto-elastic phase change method. Other methods being considered are electromechanical (E/M) impedance (Zagrai, 2007; Kruse and Zagrai, 2009), pulse-echo (Zagrai et al., 2009), and non-linear ultrasonics (Zagrai et al., 2008). These experimental studies have indicated the feasibility of using structural sensing for assessing the structural state and detecting flaws in certain cases. The methodology used in these studies has been to measure a set of pristine situations (training set) and use them as a baseline to identify changes in the signals that might be related to changes in the structural state. The work done to date has demonstrated the feasibility of such an approach (Arritt et al., 2008). However, these experiments have also revealed some implementation challenges such as:

a. Maintaining consistent baselines and keeping the sensors condition unchanged.

b. Potential confusion between changes in satellite configuration (different component placement, bolt patterns, etc.) and actual structural flaws (delaminated panels, bolts not torqued correctly, etc.).
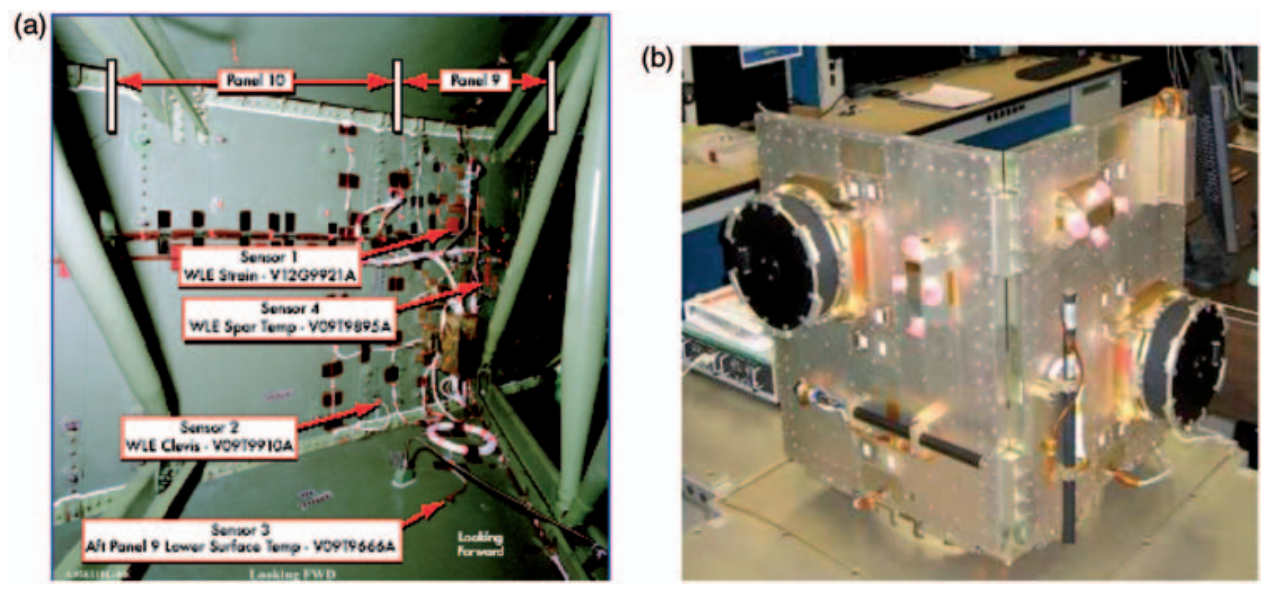

Figure 1. Potential space applications: (a) inhabited space vehicles such the NASA space shuttle (CAIB, 2003) and (b) responsive space satellites with electronics embedded into the structure (Arritt et al., 2008). 
c. Difficulty of extending outside the training set when trying to accommodate new configurations needed for new missions, orbits, payloads, technology, etc.

The need exists for developing a predictive modeling methodology to complement and assist the experimental work. Such a modeling methodology should have the capability to predict the electrical signals that would be produced by the sensors as a function of the structural state. This predictive modeling will be able to use the structural design configuration as input data and predict the sensors signals without the need of a training set or baseline. Furthermore, it will be able to predict how the signals change in the presence of structural flaws and address the correlation between sensor location and its sensitivity to a particular flaw type. The effect of variability (both in structure and in the monitoring system) could be assessed through simulation and then various statistical hypotheses could be tested. In addition, the predictive methodology would permit the testing of various detection hypotheses, as for example that the non-linearity of structural flaws could be used to separate them from changes in boundary conditions and configuration, which are supposed to have mostly linear effects.

\section{PWAS PRINCIPLES}

Piezoelectric wafer active sensors (PWAS) are the enabling technology for active SHM systems. PWAS couple the electrical and mechanical effects (mechanical strain, $S_{i j}$, mechanical stress, $T_{k l}$, electrical field, $E_{k}$, and electrical displacement $D_{j}$ ) through the tensorial piezoelectric constitutive equations:

$$
\begin{aligned}
& S_{i j}=s_{i j k l}^{E} T_{k l}+d_{k i j} E_{k} \\
& D_{j}=d_{j k l} T_{k l}+\varepsilon_{j k}^{T} E_{k}
\end{aligned}
$$

where, $s_{i j k l}^{E}$ is the mechanical compliance of the material measured at zero electric field $(E=0), \varepsilon_{j k}^{T}$ is the dielectric permittivity measured at zero mechanical stress $(T=0)$, and $d_{k i j}$ represents the piezoelectric coupling effect. PWAS utilize the $d_{31}$ coupling between in-plane strain and transverse electric field. A 7-mm diameter PWAS, $0.2-\mathrm{mm}$ thin, weighs a bare $78 \mathrm{mg}$ and costs around $\sim \$ 1$ each. PWAS are lightweight and inexpensive and hence can be deployed in large numbers on the monitored structure. Just like conventional ultrasonic transducers, PWAS utilize the piezoelectric effect to generate and receive ultrasonic waves. However, PWAS transducers are different from conventional ultrasonic transducers in several aspects:

1. PWAS transducers are small, lightweight, cheaper, and less power intensive than traditional ultrasonic transducers, which would benefit their use for space applications.

2. PWAS are firmly coupled with the structure through an adhesive bonding, whereas conventional ultrasonic transducers are weakly coupled through gel, water, or air.

3. PWAS are non-resonant devices that can be tuned selectively into several guided-wave modes, whereas conventional ultrasonic transducers are resonant narrow-band devices.

4. PWAS are inexpensive and can be deployed in large quantities on the structure, whereas conventional ultrasonic transducers are expensive and used one at a time.

By using Lamb waves in a thin-wall structure, one can detect structural anomaly, that is, cracks, corrosions, delaminations, and other damage. Because of the physical, mechanical, and piezoelectric properties of PWAS transducers, they act as both transmitters and receivers of Lamb waves traveling through the structure. Upon excitation with an electric signal, the PWAS generate Lamb waves in a thin-wall structure. The generated Lamb waves travel through the structure and are reflected or diffracted by the structural boundaries, discontinuities, and types of damage. The reflected or diffracted waves arrive at the PWAS where they are transformed into electric signals.

PWAS transducers can serve several purposes: (a) high-bandwidth strain sensors; (b) high-bandwidth wave exciters and receivers; (c) resonators; and (d) embedded modal sensors with the electromechanical (E/M) impedance method. By application types, PWAS transducers can be used for (i) active sensing of far-field damage using pulse-echo, pitch-catch, and phased-array methods, (ii) active sensing of near-field damage using high-frequency $\mathrm{E} / \mathrm{M}$ impedance method and thickness-gage mode, and (iii) passive sensing of damage-generating events through detection of lowvelocity impacts and acoustic emission at the tip of advancing cracks. Damage detection using PWAS phased arrays can detect both broadside and offside cracks independently with scanning beams emitting from a central location.

\section{PREDICTIVE MODELING OF POWER AND ENERGY TRANSDUCTION FOR SHM APPLICATIONS}

A preliminary analysis of the power and energy transduction process for SHM applications was performed (Lin and Giurgiutiu, 2010) by considering (a) PWAS transmitter; (b) PWAS receiver; and (c) PWAS transmitter-receiver pair. The electrical active power, reactive power, and power rating for harmonic voltage excitation were examined. The parametric study of transmitter size 


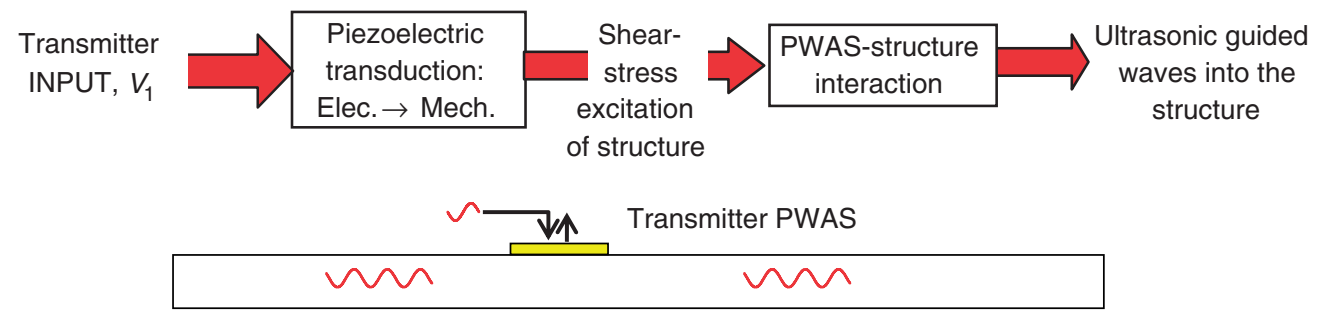

Figure 2. PWAS transmitter power and energy flow chart.

Table 1. Simulation parameters.

\begin{tabular}{|c|c|c|c|}
\hline & $\begin{array}{c}\text { Beam } \\
\text { structure } \\
\text { (2024 Al alloy) }\end{array}$ & $\begin{array}{l}\text { Transmitter } \\
\text { PWAS } \\
\text { (0.2-mm PZT) }\end{array}$ & $\begin{array}{c}\text { Receiver } \\
\text { PWAS } \\
\text { (0.2-mm PZT) }\end{array}$ \\
\hline Length & $\infty$ & $5-25 \mathrm{~mm}$ & $5-25 \mathrm{~mm}$ \\
\hline Height & $1 \mathrm{~mm}$ & $0.2 \mathrm{~mm}$ & $0.2 \mathrm{~mm}$ \\
\hline Width & \multicolumn{3}{|c|}{$40 \mathrm{~mm}$} \\
\hline Frequency & \multicolumn{3}{|c|}{ Frequency sweep 1-600 kHz } \\
\hline $\begin{array}{l}\text { Measurement } \\
\text { instrument } \\
\text { resistance }\end{array}$ & \multicolumn{3}{|c|}{$1 \Omega-1 \mathrm{M} \Omega$} \\
\hline $\begin{array}{l}\text { Constant } \\
\text { voltage input }\end{array}$ & \multicolumn{3}{|c|}{$10 \mathrm{~V}$} \\
\hline $\begin{array}{l}\text { Constant power } \\
\text { rating input }\end{array}$ & \multicolumn{3}{|c|}{$10 \mathrm{~W}$} \\
\hline
\end{tabular}

and impedance, receiver size and impedance, and external electrical load gives the PWAS design guideline for PWAS sensing and power harvesting applications. The analysis was performed in the simplifying case of axial and flexural waves, which are easier to handle than the full guided-wave model. However, the principles of this exploratory study can be extended without much difficulty to the full multi-mode guided-waves. A brief summary of this work is given next.

\section{Transmitter Power and Energy}

Figure 2 shows the power and energy transduction schematic in the case of a transmitter PWAS. The electrical energy of the input voltage applied at the PWAS terminals is converted through piezoelectric transduction into mechanical energy that activates the expansion-contraction motion of the PWAS transducer. This motion is transmitted to the underlying structure through the shear stress in the adhesive layer at the PWAS-structure interface. As a result, ultrasonic guided waves are excited into the underlying structure. The mechanical power at the interface becomes the acoustic wave power and the generated axial and flexural waves propagate in the structure. Questions that need to be answered through predictive modeling are:

(i) How much of the applied electrical energy is converted in the wave energy? (ii) How much energy is lost through the shear transfer at the PWAS-structure interface?

(iii) How much of the applied electrical energy gets rejected back into the electrical source?

(iv) What are the optimal combinations of PWAS geometry, excitation frequency, and wave mode for transmitting the maximum energy as ultrasonic waves into the structure?

To perform this analysis, Lin and Giurgiutiu (2010) developed closed-form analytical expressions for the active and reactive electrical power, mechanical power in the PWAS, and ultrasonic acoustic power of the waves traveling in the structure. The simulation considered two PWAS (a transmitter and a receiver) attached on a simple aluminum beam of infinite length. Numerical simulation was performed with the parameters given in Table 1. Constant $10-\mathrm{V}$ excitation voltage from an ideal electrical source was assumed at the transmitter PWAS. In addition, a constant power rating $10-\mathrm{W}$ source was also considered. The PWAS size was varied from 5 to $25 \mathrm{~mm}$, whereas the frequency was spanned from 1 to $600 \mathrm{kHz}$.

It was found (Figure 3) that the reactive electrical power required for the excitation of a 7-mm long PWAS is orders of magnitude larger than the active electrical power (compare Figure 3(a) with (b)) because of the inherently capacitive behavior of the PWAS; since the transmitter reactive power is directly proportional to the transmitter admittance $(Y=i \omega C)$, care must be taken to use power amplifiers that are designed to recirculate the reactive power thus reducing the overall power rating to that needed only for the excitation of structural waves which is represented by the transmitter active power, that is, the power converted into the ultrasonic acoustic waves traveling in the structure. (If conventional linear amplifiers are used, then the power rating would be dominated by the reactive power and would be much larger than needed for the ultrasonic waves alone.) The frequency variation of the active power (i.e., of the ultrasonic wave power injected into the structure) is shown in Figure 3(b); this variation is not monotonic with frequency, but manifests peaks and valleys, corresponding to tuning and then detuning between the PWAS transducer and the various ultrasonic guided waves present in the 

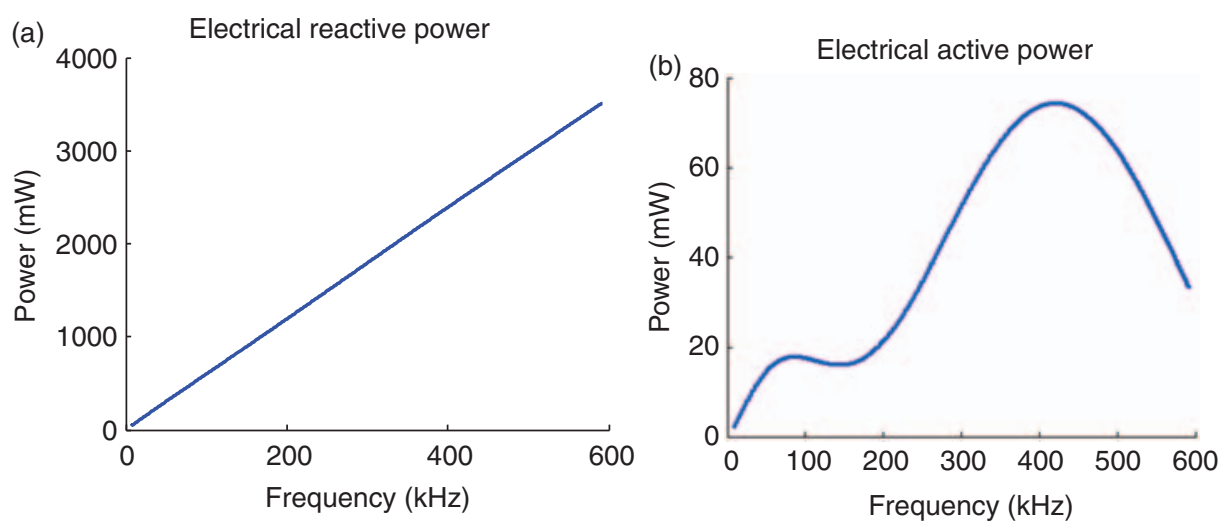

Figure 3. Electrical power required at the terminals of a 7-mm length PWAS terminals: (a) reactive power and (b) active power, i.e., the wave power injected into the structure.
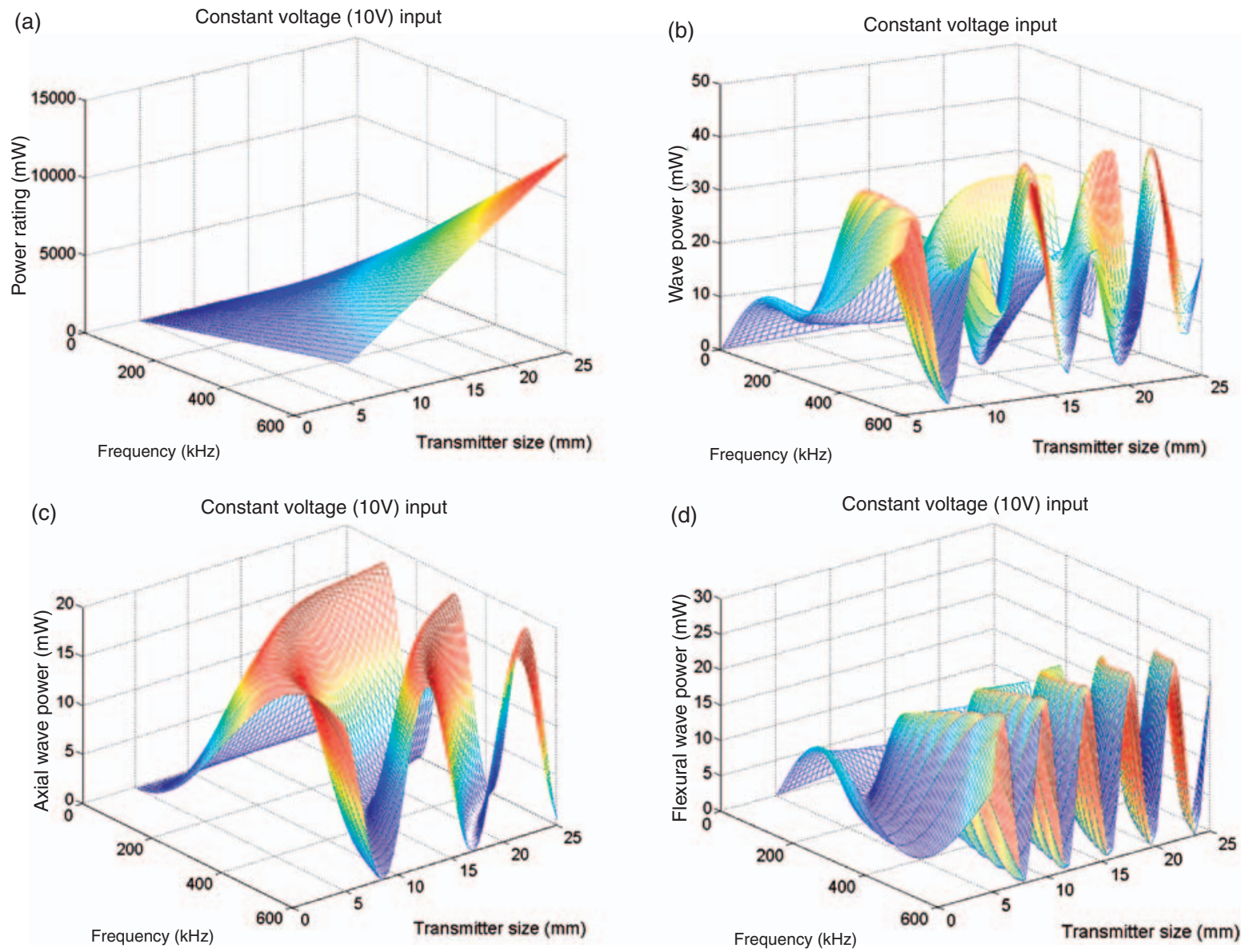

Figure 4. PWAS transmitter under constant voltage excitation: (a) power rating; (b) wave power; (c) axial wave power; and (d) flexural power.

structure. The maximum active power for a $7-\mathrm{mm}$ PWAS excited with $10-\mathrm{V}$ harmonic voltage for frequencies up to $600 \mathrm{kHz}$ seems to be $\sim 80 \mathrm{~mW}$.

Figure 4 presents the results of a parameter study for various PWAS sizes and frequencies. The resulting parameter plots are presented as 3D mesh plots. Figure 4(a) presents a 3D mesh plot of the power rating versus frequency and transmitter size: for a certain transmitter size, the power rating increases when the frequency increases. For a given frequency, the power rating increases when the transmitter size increases. These results are clarifying: to drive a $25-\mathrm{mm}$ length PWAS at $600 \mathrm{kHz}$ with a $10 \mathrm{~V}$ constant voltage input, one needs a power source providing $12.5 \mathrm{~W}$ of power. Figure 4(b) shows the wave power that PWAS generates into the structure; tuning effect of transmitter size and excitation frequency are apparent; a larger PWAS does not necessarily produce more wave power at a given 
frequency! The maximum wave power output in this simulation is $\sim 40 \mathrm{~mW}$. One notes that the wave power is about half the electrical active power; this is justified by the fact that the wave power considered here is the wave power traveling only in the forward direction. If we add the wave power traveling in both directions (forward and backward) then we get exactly the electrical active power input applied to the PWAS. Perfect electrical source and loss-less adhesive layer were assumed in this model. Future work may include source impedance and adhesive losses. This study gives guidelines for the design of transmitter size and excitation frequency in order to obtain maximum wave power into the SHM structure.
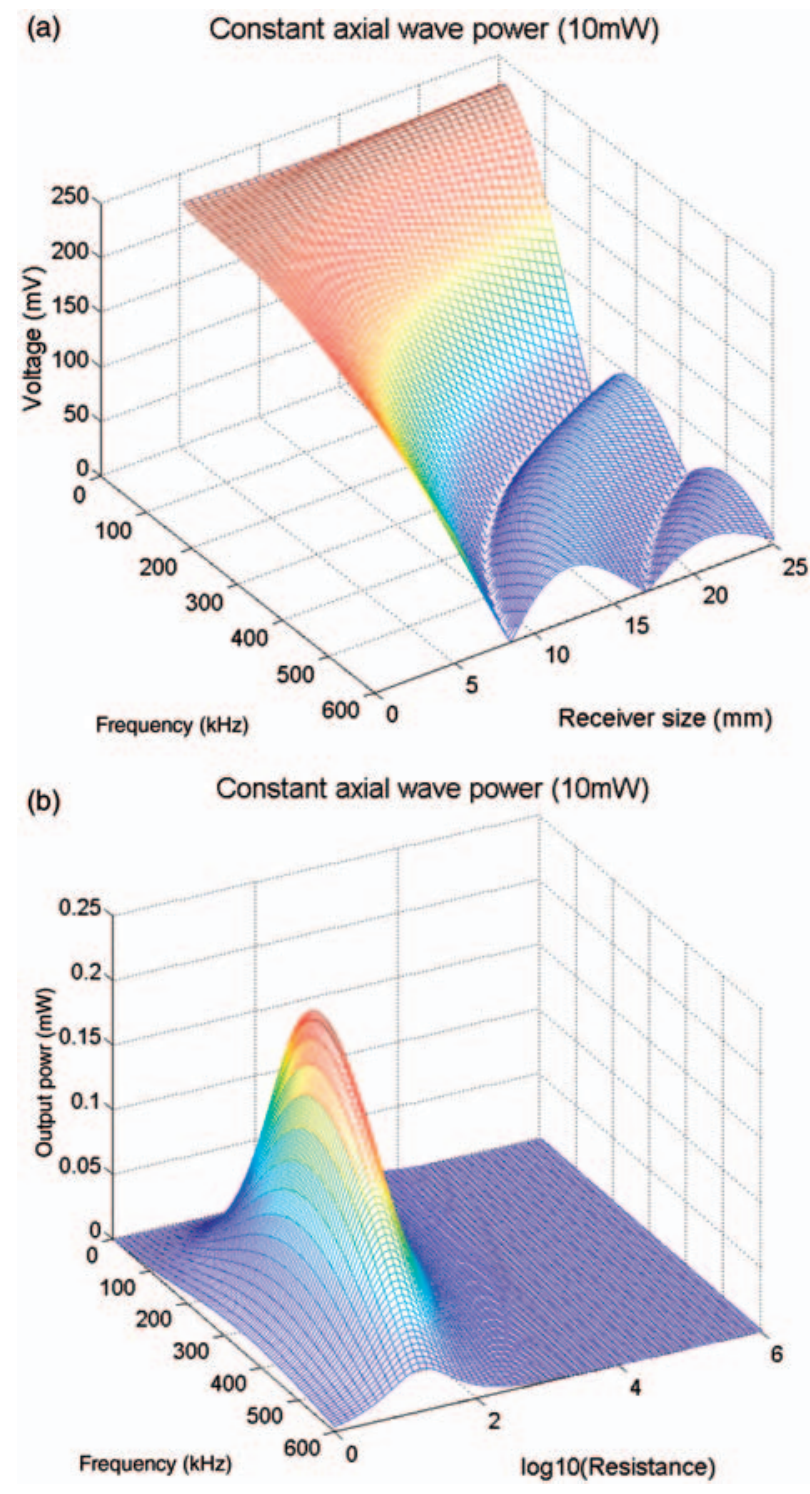

Figure 5. PWAS receiver under constant power of axial wave: (a) output voltage for sensing application and (b) Output power for power harvesting application.
The powers contained in the axial waves and flexural waves are given separately in Figure 4(c) and (d). In some PWAS SHM applications, a single mode is often desired to reduce signal complexity and simplify signal interpretation and damage detection. Figure 4(c) shows the frequency-size combinations at which the axial waves are maximized, whereas Figure 4(d) indicates the combinations that would maximize the flexural waves. These figures give useful guidelines for the choosing PWAS size and frequency values that are optimum for selecting a certain excitation wave mode. However, as power increases, attention must be paid to not exceeding the PWAS mechanical and electrical limits.

\section{Receiver Power and Energy}

A similar analysis was conducted at the receiver PWAS. The receiver PWAS was connected to an external electrical load. The external load impedance was varied between $1 \mathrm{M} \Omega$ (corresponding to an ideal measuring instrument of infinite input impedance) and $1 \Omega$ corresponding to a high-admittance energy harvester. When propagating waves reach the receiver PWAS, receiver PWAS converts the wave energy to electrical energy and outputs a voltage signal. For sensing application, a high value of the output voltage is desired. The external electrical load such as oscilloscope resistance is set to high impedance. The receiver size varies from 5 to $25 \mathrm{~mm}$ to show the sensing ability of different sensor sizes. At low frequency, the PWAS receiver shows the similar ability of sensing regardless of PWAS size. The PWAS receiver (i.e., sensing function) also shows tuning ability as we see peaks and valleys in Figure 5(a). In PWAS harvesting application, receiver size is fixed (e.g., $7 \mathrm{~mm}$ in simulation), the external electrical load impedance need to match the receiver impedance to output the maximum power. Considering a fully resistive external load varies from $1 \Omega$ to $1 \mathrm{M} \Omega$, the output electrical power is shown in Figure 5(b). The optimum resistive load for power harvesting is around $100 \Omega$ at $300 \mathrm{kHz}$ for the $7 \mathrm{~mm}$ receiver PWAS.

\section{Pitch-catch Power and Energy}

The power and energy transduction flow chart for a complete pitch-catch setup is shown in Figure 6. Under 1D assumption, the electro-acoustic power and energy transduction of the PWAS transmitter and receiver are examined. In pitch-catch mode, the power from the electrical source converts into piezoelectric power at the transmitter through piezoelectric transduction which converts the applied electrical power into mechanical power at the interface between PWAS and structure; this is further converted into ultrasonic wave power traveling in the structure. The wave power arrives at the receiver PWAS and is captured at the mechanical 


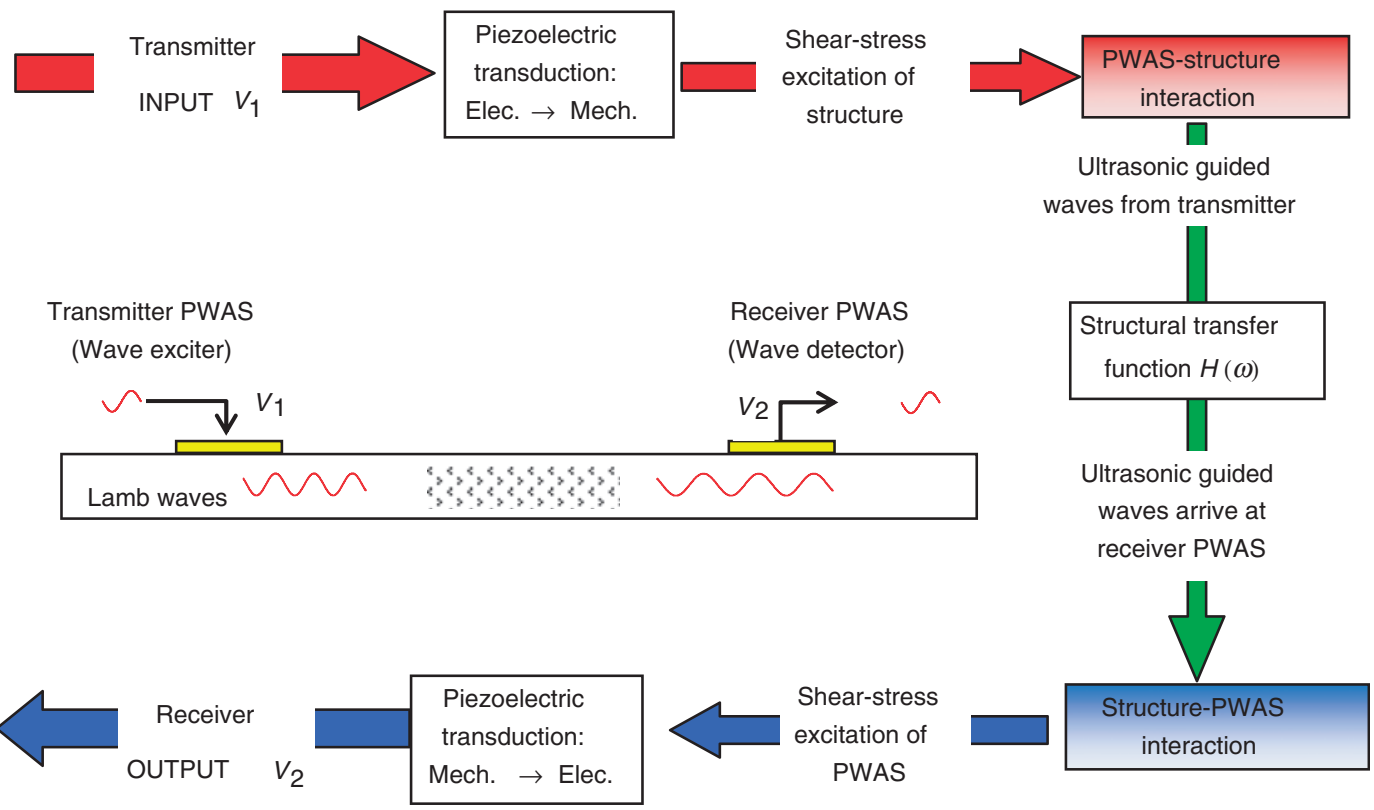

Figure 6. Power and energy flow in a PWAS pitch-catch configuration.
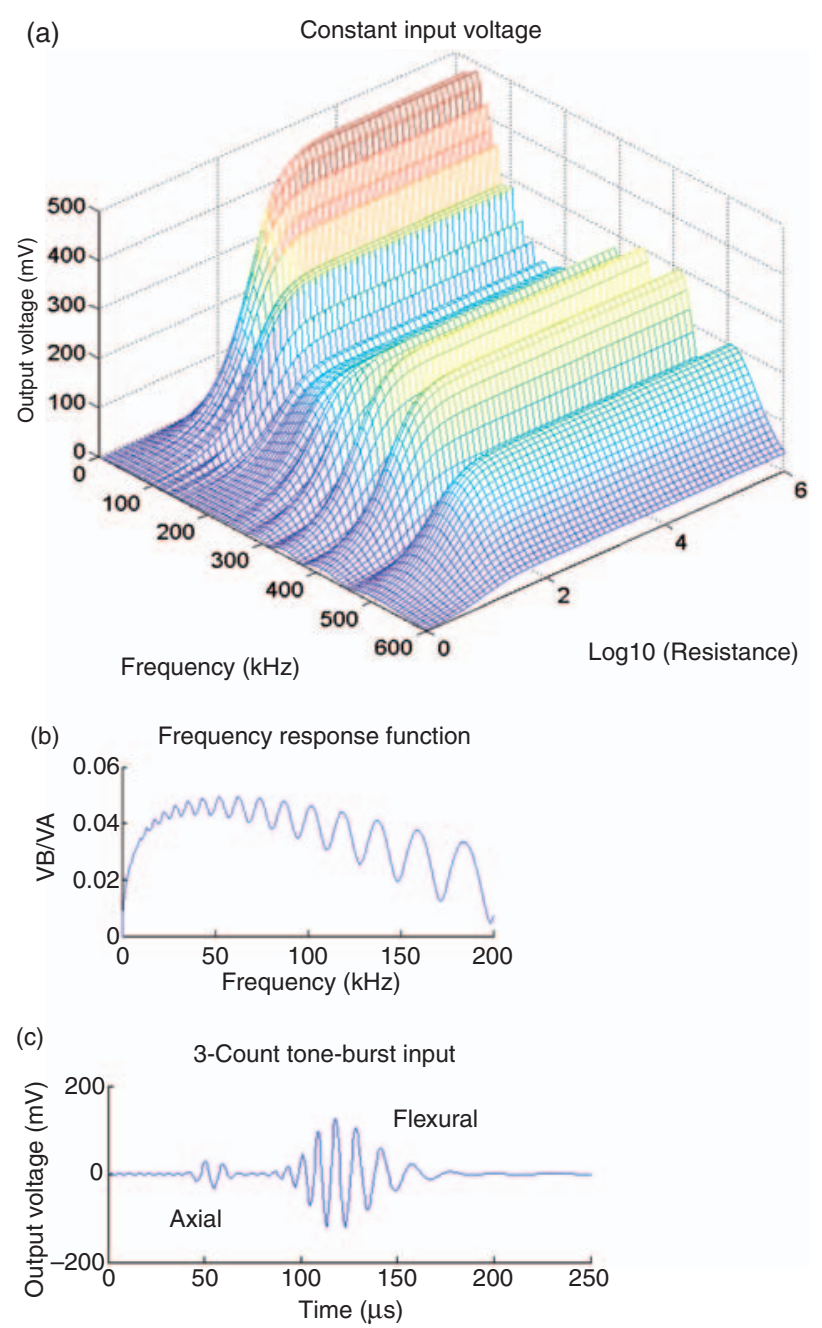

Figure 7. PWAS pitch-catch simulation under constant voltage input: (a) output voltage in relation of output resistance; (b) voltage output under harmonic excitation; and (c) voltage under tone-burst excitation. interface between the receiver PWAS and the structure. The captured mechanical power is converted back into electrical power through the piezoelectric effect in the receiver PWAS and read as voltage signal by the receiver electrical instrument. The time-averaged electrical power, mechanical power at the transmitter and wave power can be calculated from the frequency response function. The time-averaged mechanical power and electrical power at the receiver PWAS can be calculated as well.

In pitch-catch setup simulation, an Aluminum alloy 2024 infinite bar was used with $40 \mathrm{~mm}$ width and $1 \mathrm{~mm}$ thickness. PWAS transmitter and receiver are 7-mm length, 40-mm width and 0.2-mm thickness. A $10 \mathrm{~V}$ harmonic voltage is applied on the transmitter PWAS. The distance between transmitter and receiver is $200 \mathrm{~mm}$.

Figure 7(a) shows the output voltage of receiver PWAS when transmitter PWAS is excited by a harmonic constant amplitude $(10 \mathrm{~V})$ input. The output electrical load varies. Figure 7(b) shows the frequency response function when the output electrical load is set at high Impedance (1 M $\Omega$ ). A $100-\mathrm{kHz}$ central frequency 3-count Hanning window tone-burst signal is applied to the transmitter. The receiver instantaneous voltage response is shown in Figure 7(c). The fast axial wave is separated from the low speed flexural wave. The axial wave is non-dispersive and keeps the shape of excitation signal. The flexural wave spread out due to the dispersive nature.

\section{PWAS SHM EXPERIMENTS ON A SPACECRAFT-LIKE PANEL SPECIMEN}

The possibility of using ultrasonic PWAS transducers for structural health monitoring space applications was investigated on a metallic spacecraft specimen (Cuc et al., 2007). The panels consist of the skin (Al 7075, 


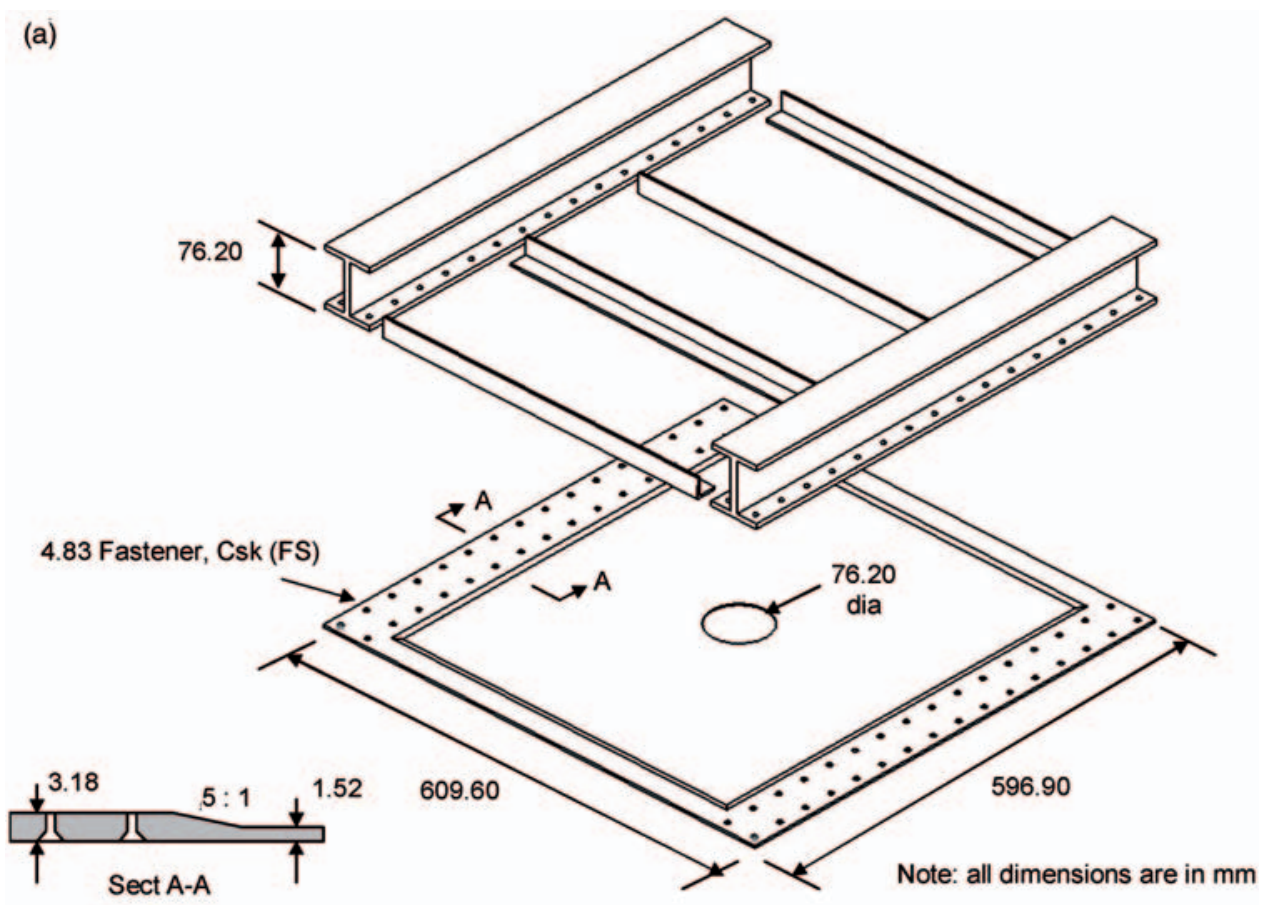

(b)

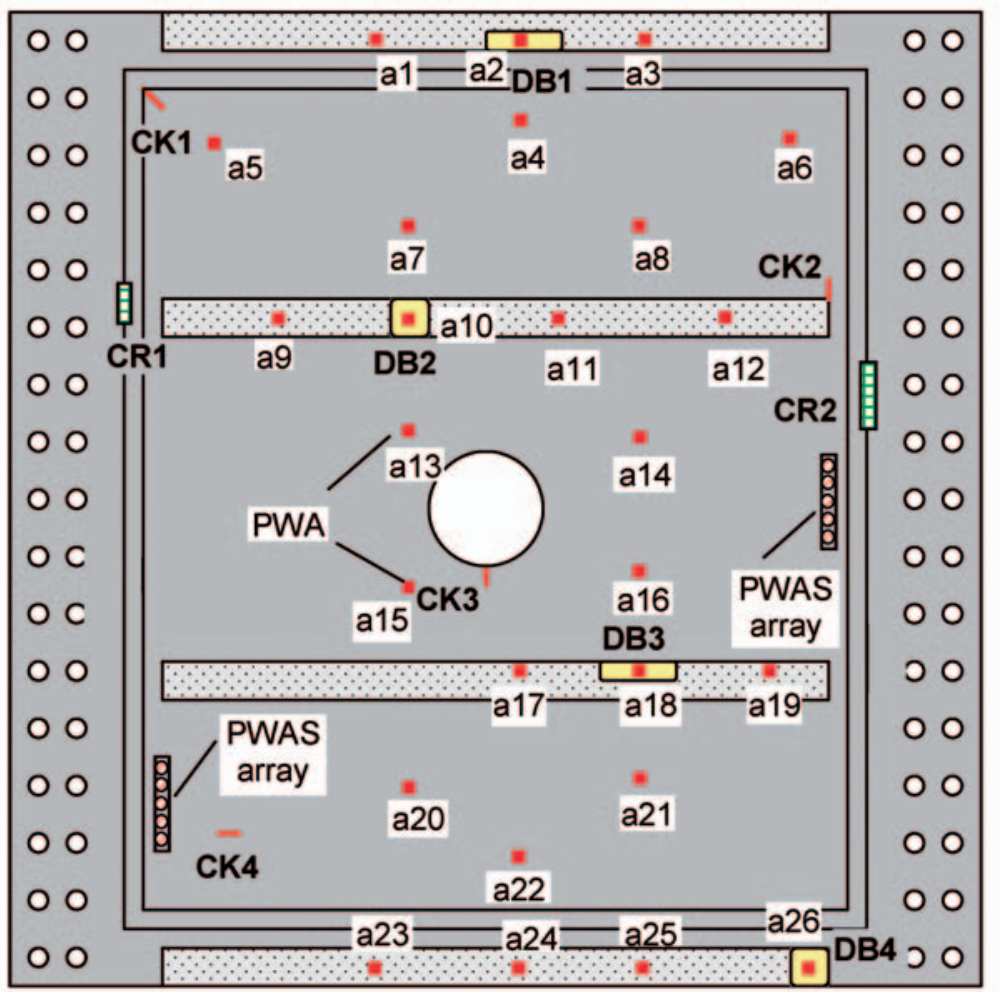

Figure 8. Spacecraft like panel specimen: (a) overall layout and (b) PWAS and damage location.

$609.60 \mathrm{~mm} \times 596.90 \mathrm{~mm} \times 3.18 \mathrm{~mm})$ with a $76.2 \mathrm{~mm}$ diameter hole in the center, two spars (Al 6061 I-beams, $\quad 76.20 \mathrm{~mm} \times 63.50 \mathrm{~mm} \times 6.35 \mathrm{~mm}$ and $609.60 \mathrm{~mm}$ length), four stiffeners (Al 6063, $25.40 \mathrm{~mm} \times$ $25.40 \mathrm{~mm} \times 3.18 \mathrm{~mm}$ and $469.90 \mathrm{~mm}$ length) and fasteners installed from the skin side (Figure 8(a)).
The stiffeners were bonded to the aluminum skin using a structural adhesive, Hysol EA 9394. Damages were artificially introduced in the two specimens including cracks (CK), corrosions (CR), disbonds (DB), and cracks under bolts (CB). A schematic of the aluminum Panel 1 specimen showing the location of the damage is presented in 


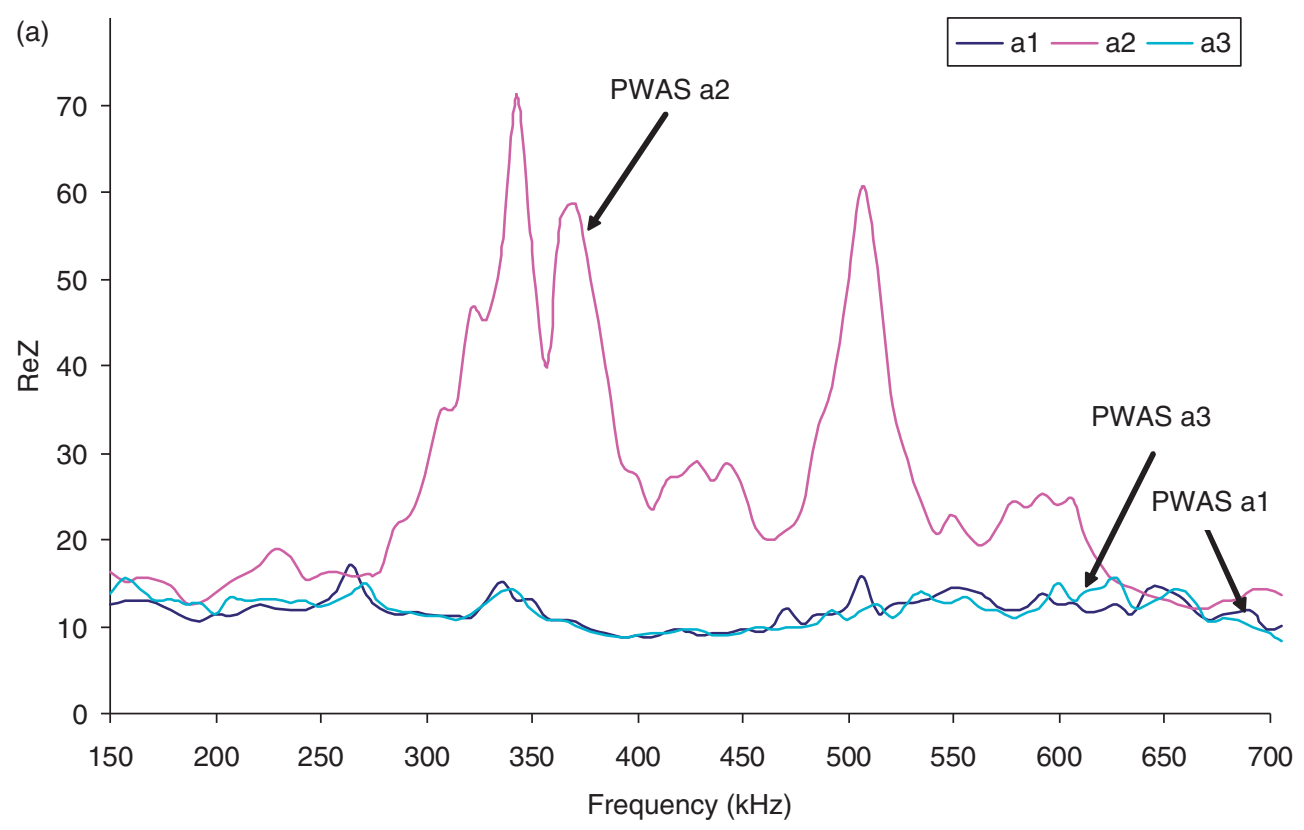

Free PZT PWAS

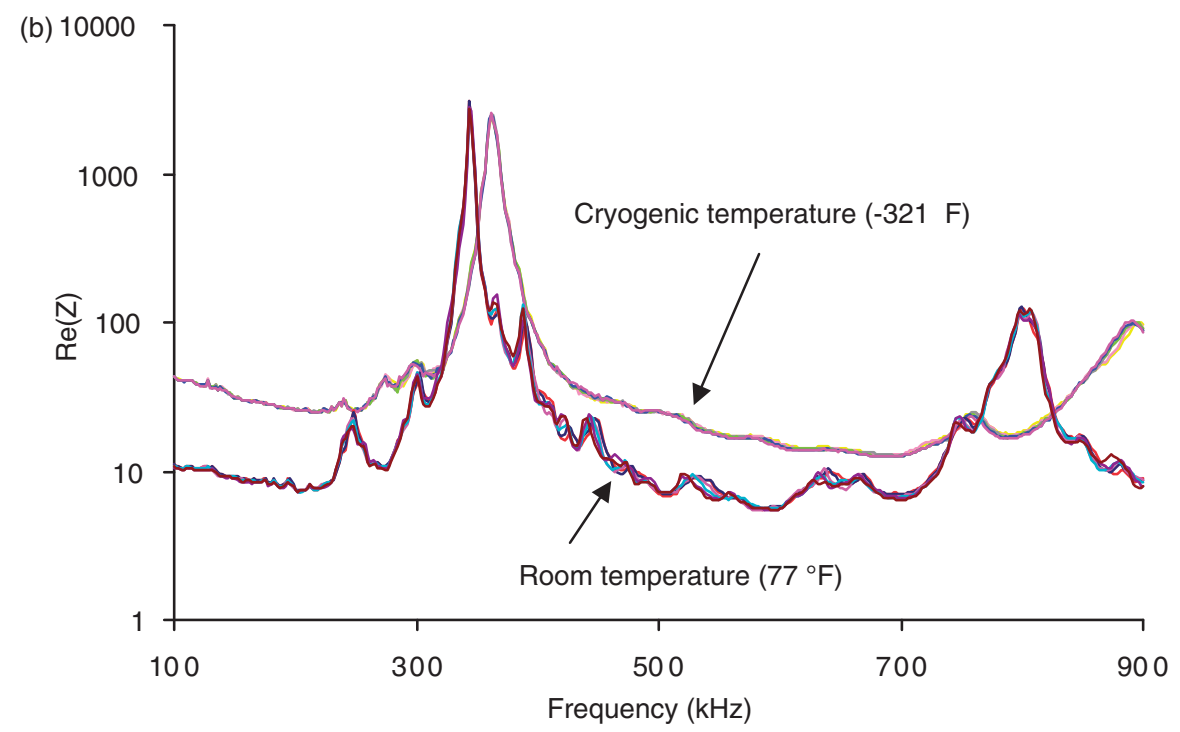

Figure 9. E/M impedance method applied in spacecraft conditions: (a) resonant frequencies spectrum showing increased amplitude for the signal received at the sensor located on the top of disbond DB1 (PWAS a2 of Figure 8(b)) and (b) cryogenic test of a free PWAS in liquid nitrogen showing sustained activity and spectrum shifts.

Figure 8(b). Panel 1 contains disbonds, cracks and corrosions. The disbonds are located between the stiffeners and the skin. They are of two types: partial disbonds DB1 and DB3, and complete disbonds DB2 and DB4. The corrosions are simulated as machined areas were part of material was removed. The four cracks presented are in the shape of a slit and are through cracks located on the skin of the panel. The two panels were instrumented with PWAS as shown in Figure 8(b). The PWAS were used for both sending and receiving Lamb waves. The location and the number of sensors depend on the detection method. We used wave propagation and standing wave damage detection methods as follows: pitch-catch for disbond detection; pulse-echo for disbond and crack detection; embedded ultrasonic structural radar (EUSR) for crack detection; electromechanical (E/M) impedance for disbond, crack, and corrosion detection. For illustration, Figure 9(a) presents the E/M impedance spectrum measured on PWAS a2 (above a disbond) in comparison with that of PWAS a1 and a3 which were on well bonded regions. It is apparent that (i) the $\mathrm{E} / \mathrm{M}$ impedance spectra are consistent between a1 and a3; and (ii) the presence of a disbond drastically changes the $\mathrm{E} / \mathrm{M}$ impedance. Full details of all the tests performed and of the corresponding results can be found in Cuc et al. (2007). 


\section{PWAS OPERATION EXPERIMENTS AT CRYOGENIC TEMPERATURES}

The use of PWAS transducers for spacecraft applications raises new issues related to the in-space environmental conditions, namely operation at cryogenic temperatures. We did a series of experiments to verify that PWAS transducers can operate at cryogenic temperatures and what measures must be taken to ensure their survivability in these conditions (Lin et al., 2010). The experiments were conducted after a careful selection of the adhesive layer between the PWAS and the structure and solder material between PWAS and electric wire. A 2-component adhesive (Vishay M-Bond AE15) was selected. The indium-based solder had to be used because the usual $\mathrm{Sn} / \mathrm{Pb}$ solder becomes brittle at cryogenic temperatures. Free and bonded PWAS were submerged into liquid nitrogen $(-21 \mathrm{~F},-196 \mathrm{C})$, soaked for 10 min and then measured with an HP 4194A impedance analyzer. No major E/M impedance changes were recorded for both free and bonded PWAS working at cryogenic temperature. The results shown in Figure 9(b) indicate that a free PWAS continues to resonate when subjected to cryogenic temperatures in liquid nitrogen but the resonance frequency increased from 344 to 362 $\mathrm{kHz}$. However, the initial impedance signature was recovered when the PWAS was warmed back to room temperature. The results for PWAS bonded to circular aluminum plates are shown in Figure 10. These results indicate that a PWAS bonded to a circular aluminum (a)

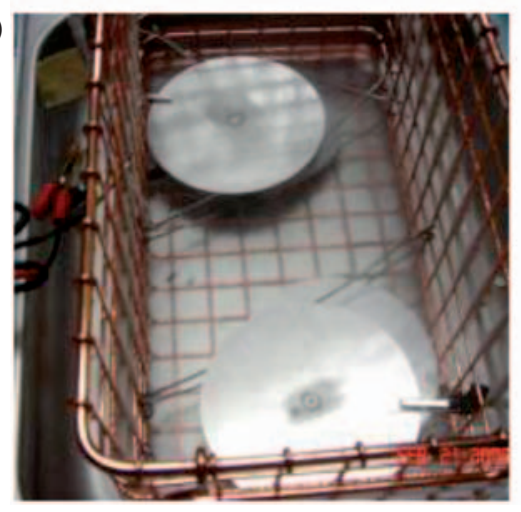

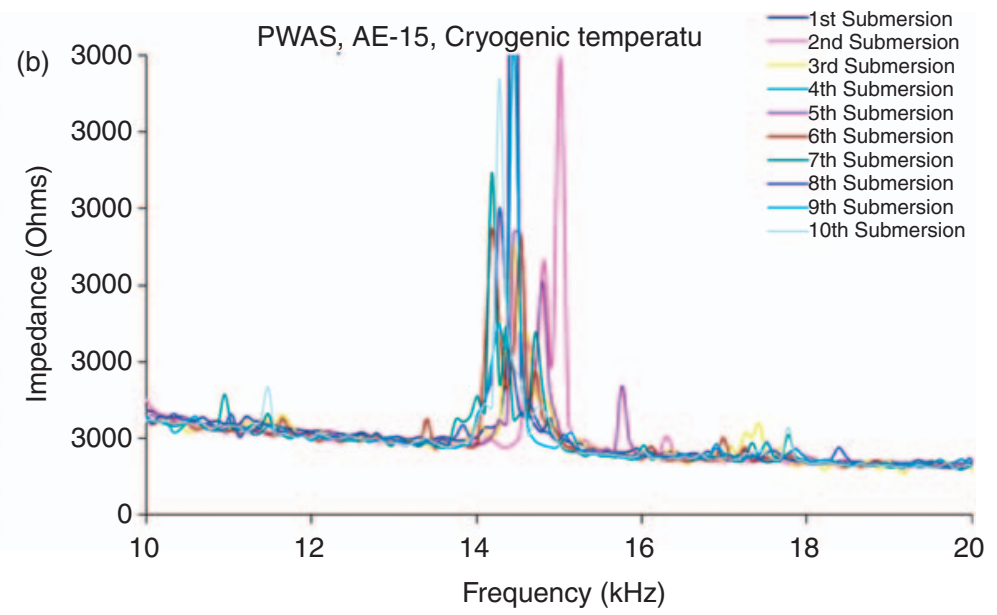

Figure 10. The real part of impedance indication of operability through retention of resonant properties while submersed in liquid nitrogen: (a) experimental setup and (b) E/M impedance curves.

(a)

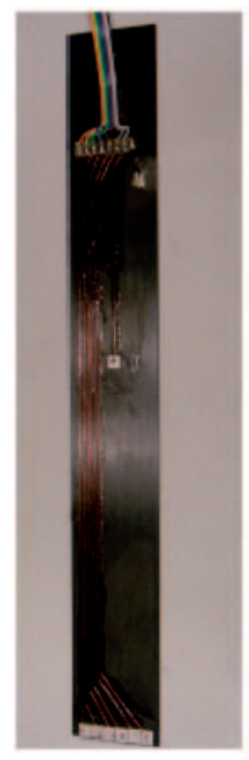

(b)

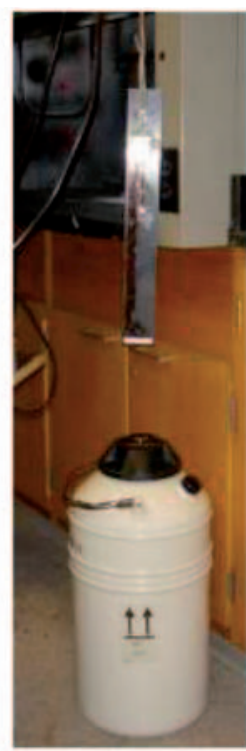

(c) 25
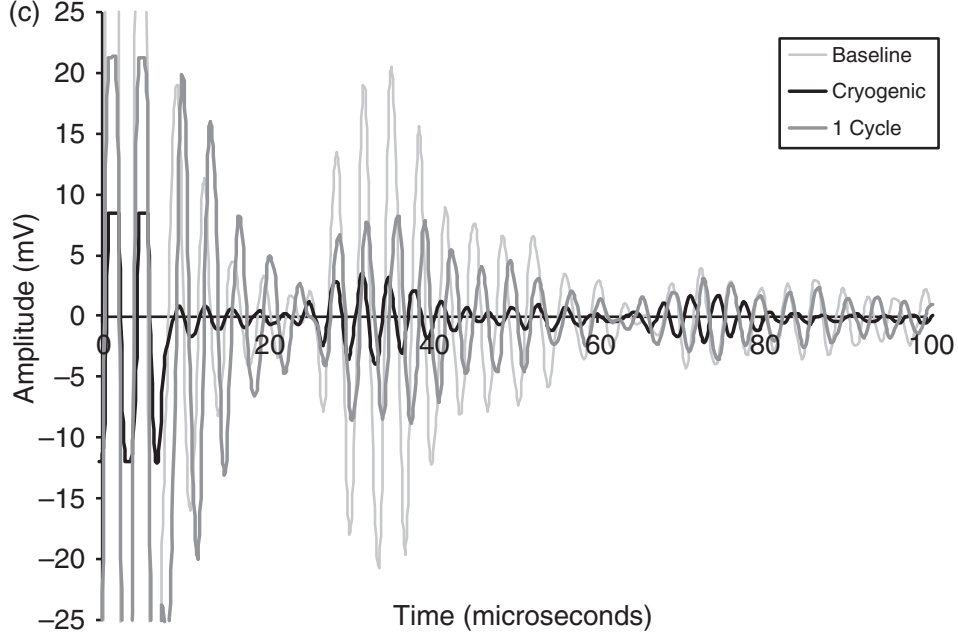

Figure 11. Pitch-catch experiments at cryogenic temperature: (a) unidirectional carbon-fiber composite specimen; (b) experimental set-up shown the specimen on the way of being submersed in liquid nitrogen tank; and (c) wave packet before, during, and after submersion in liquid nitrogen. 
plate retains its operability while submerged in liquid nitrogen (Figure 10(b)).

A unidirectional carbon fiber composite strip (Figure 11(a)) was used to determine if the PWAS are able to send and receive guided waves at cryogenic temperatures. Figure 11(b) shows the experimental set-up and Figure 11(c) shows the pitch-catch wave signal. One notices that, when the specimen was submerged in liquid nitrogen, the amplitude of the wave packet decreased. After returning to room temperature, the amplitude of the wave packets did not return to the original amplitudes; we believe that the adhesive interface was affected by liquid nitrogen submersion. Further research is needed to determine the cause of this phenomenon; we believe that this issue can be corrected by using adhesive formulations that are qualified for extreme temperatures. The wave packets amplitudes were greater than when submerged in liquid nitrogen; this may be due to the fact that while the specimen was in liquid nitrogen, the wave excited by the PWAS leaked into the liquid. In future work, adhesive formulations better suited for extreme temperatures should be used. In addition, the cooling of the specimen should be achieved in a different way such that direct contact to the liquid nitrogen is avoided and wave energy leaking in the fluid is prevented; these experimental conditions would represent more adequately the actual space operation conditions.

\section{CONCLUSIONS}

This article has presented and discussed the challenges and opportunities related to the use of PWAS in structures specific to space applications. The article started with a brief presentation of the challenges posed by space structures, which are often different from those encountered in conventional structures. Responsive space requirements for accelerated structural surety were shown to offer important SHM opportunities. A review of PWAS-based SHM principles was given. A discussion of predictive modeling of the multi-physics power and energy transduction between structurally guided waves and PWAS followed. Predictive modeling results using a simplified analytical approach were presented and discussed. It was shown that a judicious combination of PWAS size, structural thickness, and excitation frequency can ensure optimal energy transduction and coupling with the ultrasonic guided waves traveling in the structure. Experimental results on using PWAS technology to detect damage in space-like specimen structures were presented next. A spacecraft-like specimen containing seeded crack, corrosion, and disbonds was tested with a battery of PWAS based SHM methods (pitch-catch, pulse-echo, phased-arrays, E/M impedance). An example of disbond detection with the $\mathrm{E} / \mathrm{M}$ impedance method was presented. The operability and survivability of PWAS transducers under cryogenic space-like conditions was experimentally verified. It was shown that PWAS transducers can operate in liquid nitrogen at $-321 \mathrm{~F}(-196 \mathrm{C})$. E/M impedance spectrum showed slight shifts which were reversed upon return to room temperature. Pitch-catch experiments conducted on unidirectional carbon fiber composite specimens indicated that submersion in liquid nitrogen diminishes the signal strength, which may be due to waves leakage. The initial response was not recovered upon return to room temperature, which may indicate adhesion problems. Our test specimen is more specific to inhabitable space vehicle structures (e.g., international space station, orbital space plane) rather than uninhabited satellites. The results of our work are relevant to the larger space structures community. Further research is needed to better understand the interaction of guided waves with damage in spacecraft structures and how they would survive in the harsh space environment. Development of a predictive model to optimize the sensor-structure configuration for effective damage detection with minimum weight and power requirement on the SHM system should be also pursued.

\section{ACKNOWLEDGMENTS}

This work was partially supported by NASA STTR T7-02 through NextGen Aeronautics, Inc. and by the National Science Foundation award CMS-0925466.

\section{REFERENCES}

Arritt, B.J., Buckley, S.J., Ganley, J.M., Kumar, A., Clayton, E.H., Hannum, R., Todd, M.D., Kennel, M.B., Welsh, J., Beard, S., Stabb, M.C., Xinlin, Q. and Wegner, P. 2007. "Responsive Satellites and the Need for Structural Health Monitoring," In: SPIE, Vol. 6531:6531-6609.

Arritt, B.J., Robertson, L.M., Williams, A.D., Henderson, B.K., Buckley, S.J., Ganley, J.M., Welsh, J.S., Ouyang, L., Beard, S., Clayton, E.H., Todd, M.D., Doyle, D. and Zagrai, A.N. 2008. "Structural Health Monitoring: an Enabler for Responsive Satellites," In: 49th AIAA Structural, Structural Dynamics, and Materials Conference, Schaumburg, IL, 7-10 April 2008, \#AIAA-2008-2166.

CAIB. 2003. "Columbia Accident Investigation Report Volume I", August 2003, Columbia Accident Investigation Board, NASA, http://www.nasa.gov/columbia/caib/html/start.html.

Clayton, E.H., Kennel, M.B., Fasel, T.R., Todd, M.D., Stabb, M.C. and Arritt, B.J. 2008. "Active Ultrasonic Joint Integrity Adjudication for Real-time Structural Health Monitoring," In: SPIE, Vol. 6935:1-11. 69350M.

Cuc, A., Giurgiutiu, V., Joshi, S. and Tidwell, Z. 2007. "Structural Health Monitoring with Piezoelectric Wafer Active Sensors for Space Applications," AIAA Journal, 45:2838-2850.

Doyle, D., Zagrai, A. and Arritt, B. 2009. "Bolted Joint Integrity for Structural Health Monitoring of Responsive Space Satellites," In: 50th AIAA/ASME/ASCE/AHS/ASC Structures, Structural Dynamics, and Materials Conference, Palm Springs, California, 4-7 May 2009, Paper \#AIAA 2009-2705.

Doyle, D., Zagrai, A., Arritt, B.J. and Çakan, H. 2008. "Damage Detection in Satellite Bolted Joints," In: 2008 ASME SMASIS 
Conference on Smart Materials, Adaptive Structures and Intelligent Systems, Ellicott City, Maryland, Paper, 28-30 October, 2008, SMASIS2008-550.

Giurgiutiu, V. 2008. Structural Health Monitoring with Piezoelectric Wafer Active Sensors, 760 Elsevier Academic Press.

Kruse, W. and Zagrai, A.N. 2009. "Investigation of Linear and Nonlinear Electromechanical Impedance Techniques for Detection of Fatigue Damage in Aerospace Materials," 7th International Workshop on Structural Health Monitoring, edn. September 2009, pp. 9-11, Stanford University, CA.

Lin, B. and Giurgiutiu, V. 2010. "Modeling of Power and Energy Transduction of Embedded Piezoelectric Wafer Active Sensors for Structural Health Monitoring," In: SPIE, Vol. 7647, Paper \#7647-7697.

Lin, B., Giurgiutiu, V., Pollock, P., Xu, B. and Doane, J. "2010. "Durability and Survivability of Piezoelectric Wafer Active Sensors on Metallic Structure," AIAA Journal, 48:635-643.

Sarafin, T.P. and Doukas, P.G. 2007. "Simplifying the Structural Verification Profess to Accommodate Responsive Launch," In: 5th Responsive Space Conference, Los Angeles, CA, 23-26 April 2007, Paper \# AIAA RS5-2007-5003.

Sega, R.M. and Cartwright, J.E. 2007. "Plan for Operationally Responsive Space; A Report to Congressional Defense
Committees," National Security Space Office, 17 April 2007,http://www.responsivespace.com/Conferences/RS5/4=17= 07\%20ORS\%20Plan.pdf.

Xie, W., Zhang, B., Du, S. and Dai, F. 2007. "Experimental Investigation of Bolt Loosening Detection in Thermal Protection Panels at High Temperatures," 6th International Workshop on Structural Health Monitoring, Vol. 2, pp. 1480-1490, Stanford, CA.

Yang, J. and Chang, F.-K. 2006. "Detection of Bolt Loosening in C-C Composite Thermal Protection Panels: Part II - Experimental Verification," Smart Materials and Structures, 15:591-599.

Zagrai, A. 2007. "Electro-Mechanical Analogies for Modeling the Structural Impedance Response," In: SPIE, Vol. 6532, Paper \# 6532-14.

Zagrai, A., Doyle, D. and Arritt, B. 2008. "Embedded Nonlinear Ultrasonics for Structural Health Monitoring of Satellite Joints," In: SPIE, Vol. 6935, Paper \# 693505.

Zagrai, A., Doyle, D., Gigineishvili, V., Gardenier, H., Brown, J. and Arritt, B. 2009. "Developing a Piezoelectric Active Sensor SHM System for Satellites," In: 2009 ASME SMASIS Conference on Smart Materials, Adaptive Structures, and Intelligent Systems Conference, Oxnard, CA, 21-23 September, 2009, Paper \# SMASIS2009-1342. 